\title{
Influência da prática do CrossFit $₫$ sobre a função muscular do assoalho pélvico em mulheres
}

\author{
Influence of CrossFit ${ }^{\circledR}$ Practice on Pelvic \\ Floor Muscle Function in Women
}

Ketiane Moura Bezerra ${ }^{\mathrm{I}}$ Rainara dos Santos Monteiro Thiffany da Costa DA Costa LIMA $^{I}$

Rafaela Cordeiro de Macêdo ${ }^{\mathrm{I}}$ Centro Universitário do Estado do Pará (CESUPA), Belém/PA - Brasil.
Resumo - Introdução: O CrossFit ${ }^{\circledast}$ é uma atividade física com movimentos de alta intensidade que causam sobrecarga na musculatura perineal, tornando-se um fator de risco para o surgimento de disfunções do assoalho pélvico. Objetivo: Avaliar a influência da prática do CrossFit ${ }^{\text {t }}$ sobre a função muscular do assoalho pélvico em mulheres. Métodos: Trata-se de um estudo observacional, descritivo e quantitativo com mulheres entre 19 e 40 anos, a avaliação funcional do assoalho pélvico foi feita por meio do esquema PERFECT e da função sexual pelo questionário Female Sexual Function Index (FSFI). Resultados: Participaram da pesquisa 32 mulheres, com média de idade de 25,56 anos, o tempo médio de prática do CrossFit $^{\oplus}$ foi de 18,28 meses. Quanto à perda de urina, $75 \%$ delas relataram perda de urina durante a execução dos exercícios, sendo esses o Double Under, Box Jump, Medicine-Ball Clean e Kettlebell Swing. Quanto ao PERFECT usado para avaliar a contração da musculatura do assoalho pélvico (MAP), demostrou-se baixo em todas as variáveis e no escore total do FSFI com valor $<26$, expressando alto risco de disfunção sexual. Conclusão: $\mathrm{O}$ CrossFit ${ }^{\circledR}$ demonstrou influenciar a funcionalidade do MAP em mulheres, fatores como o tipo de parto vaginal e o número de gestações contribuíram para desencadear a incontinência urinária durante a prática do esporte, favorecendo a redução da força do MAP e apontando influência sobre a função sexual.

Palavras-Chave: Fisioterapia; Exercício; Assoalho pélvico.

\begin{abstract}
Introduction: CrossFit ${ }^{\oplus}$ is a physical activity with high intensity movements that overload the perineal muscles, becoming a risk factor for the emergence of pelvic floor disorders. Objective: To evaluate the influence of CrossFit $^{\oplus}$ practice on pelvic floor muscle function in women. Methods: This is an observational, descriptive and quantitative study with women between 19 and 40 years old, the functional assessment of the pelvic floor was performed using the PERFECT scheme and sexual function using the Female Sexual Function Index (FSFI) questionnaire. Results: 32 women participated in the research, with an average age of 25.56 years, the average time of practice of CrossFit ${ }^{\oplus}$ was 18.28 months. As for urine loss, $75 \%$ of them reported urine loss during the execution of the exercises, which are the Double Under, Box Jump, Medicine-Ball Clean and Kettlebell Swing. As for the PERFECT used to assess pelvic floor muscle contraction, it was low in all variables and in the total FSFI score with a value $<26$, expressing a high risk of sexual dysfunction. Conclusion: CrossFit ${ }^{\circ}$ has been shown to influence the functionality of the pelvic floor muscle in women, factors such as the type of vaginal delivery and the number of pregnancies contributed to triggering urinary incontinence during the practice of sports, favoring the reduction of pelvic floor muscle strength and indicating an influence on the function sexual Keywords: Physiotherapy; Exercise; Pelvic Floor.
\end{abstract}




\section{INTRODUÇÃO}

As disfunções do assoalho pélvico (DAP) são responsáveis por aproximadamente 300.000 procedimentos cirúrgicos anualmente nos Estados Unidos. ${ }^{1}$ A DAP é definida como uma condição ginecológica relacionada ao comprometimento na integridade dessa musculatura, englobando prolapsos dos órgãos pélvicos, disfunções sexuais, incontinência urinária e anal ${ }^{1,2,3}$

Dentre essas disfunções, a incontinência urinária (IU) é definida segundo a Sociedade Internacional de Continência (International Continence Society - ICS) como a queixa de qualquer perda involuntária de urina. ${ }^{4}$ Já o prolapso constitui-se em uma herniação dos órgãos pélvicos através da vagina, representando um problema socioeconômico importante, ${ }^{5,6}$ especialmente em relação à disfunção sexual feminina, essa é definida como qualquer desordem relacionada ao desejo sexual, excitabilidade, orgasmo ou dor sexual, sendo capaz de influenciar a saúde física e mental e pode ser afetada por fatores orgânicos, emocionais e sociais. ${ }^{7,8}$

Do ponto de vista biomecânico, o assoalho pélvico é responsável pela continência, sustentação dos órgãos pélvicos e da resposta sexual, sendo sua estrutura anatômica composta por fáscias pélvicas (ligamento pubo-vesical, redondo do útero, uterossacro e ligamento cervical transverso), o diafragma pélvico (puborretal, pubococcígeo, iliococcígeo e coccígeo) e o diafragma urogenital (músculo bulboesponjoso, transverso superficial, isquiocavernoso e esfíncter externos), e fibras musculares $70 \%$ do tipo I (lenta) e 30\% do tipo II (rápida)., ${ }^{9} 10$
Segundo Almeida et al. (2015) ${ }^{11}$, atividades físicas de alto impacto podem contribuir entre os fatores que possibilitam as maiores queixas de perda de urina, perda de gás e sintomas sexuais entre as mulheres que praticam esportes de alto impacto ou de forte esforço, o que se justifica o aumento da pressão intra-abdominal durante os exercícios e, devido a esse aumento crônico durante o esporte, é mais provável que a perda de urina ocorra. Alguns autores destacam que essas atletas apresentam sobrecarga, estiramento e enfraquecimento do assoalho pélvico; outra justificativa exposta seria que os distúrbios endócrinos influenciam nas DAP, a amenorreia hipotalâmica decorrente de exercício físico intenso e as desordens alimentares ou a combinação de ambos, também poderia contribuir para a IU em atletas em razão aos baixos níveis de estrogênios. ${ }^{12}$

O CrossFit ${ }^{\oplus}$ é considerado uma atividade vigorosa que inclui movimentos funcionais de alta intensidade e de variações constantes como levantamento olímpico, movimentos ginásticos e de condicionamento aeróbio, seus exercícios abordam três aspectos principais: suportar altas cargas, percorrer grandes distâncias e executar movimentos em alta velocidade, que podem oferecer um alto risco de lesão ao praticante. Ainda por se tratar de exercícios nos quais há uma grande transmissão de impacto que acontece entre os pés e o chão causando uma sobrecarga para o assoalho pélvico. ${ }^{13,14,15}$

Antunes et al $(2011)^{16}$ ressaltam que a prática de atividade física está difundida como uma das formas de manter hábitos saudáveis de vida, e indivíduos que apresentam DAP podem deixar de praticar exercícios devido aos incômodos e aos sintomas 
dessas disfunções. Dessa forma, as DAP podem comprometer a concentração, o desempenho, a execução dos gestos esportivos, restringir a hidratação e até mesmo induzir ao abandono do esporte. ${ }^{12}$

Pelo exposto, a pesquisa objetivou avaliar a influência da prática do CrossFit ${ }^{\circledR}$ sobre a função muscular do assoalho pélvico em mulheres.

\section{Procedimentos METODOLÓGICOS}

A pesquisa caracterizou-se como um estudo observacional, descritivo e quantitativo. Realizada na Clínica Escola de Fisioterapia do Centro Universitário do Estado do Pará, aprovado pelo Comitê de Ética em Pesquisa da mesma instituição sob o parecer número 3.501.120. Todas as avaliações foram realizadas pela mesma pesquisadora no período de setembro a outubro de 2019. A amostra foi voluntária, composta por 32 mulheres, sendo recrutadas por meio de convite verbal e divulgação de material informativo em redes sociais e nas próprias academias. Todas participaram de uma entrevista entes de iniciar a avaliação para identificar se as mulheres se enquadravam nos critérios de inclusão e exclusão. Os critérios para participar do estudo foram: mulheres entre 18 a 45 anos, praticantes de CrossFit $^{\circ}$ de 07 academias de Belém do Pará que realizavam a prática do esporte de no mínimo duas vezes por semana, por mais de seis meses, tendo iniciado vida sexual e sem diagnóstico de disfunção do assoalho pélvico antes de iniciarem a prática de CrossFit ${ }^{\oplus}$. Foram excluídas mulheres que realizaram intervenções cirúrgicas ginecológicas no período de um ano, que realizam outro tipo de atividade física de grande intensidade, tratamento fisioterapêutico uroginecológico, mulheres grávidas, partos recentes e multiparidade (tendo no máximo cinco filhos $)^{17}$, história de incontinência urinária antes da prática do CrossFit e que apresentam algum déficit cognitivo. Todas as mulheres que expressaram interesse em participar da pesquisa manifestaram-se por meio da assinatura do Termo de Consentimento Livre e Esclarecido (TCLE).

Inicialmente foi realizada uma anamnese a fim de caracterizar a amostra com dados sociodemográficos, histórico ginecológico e questões adicionais a respeito do histórico esportivo e a ocorrência de perda de urina durante a prática do esporte. Além da avaliação da função sexual por meio do questionário FSFI que foi desenvolvido e validado nos Estados Unidos, e traduzido para a língua portuguesa, o qual é composto por 19 questões que abordam: desejo sexual, excitação, lubrificação, orgasmo, satisfação e dor. A partir de cada resposta é atribuído um valor de 0 a 5 , então, realiza-se um cálculo matemático para a obtenção do escore total do FSFI. O formulário visa identificar possíveis alterações sexuais com o objetivo de avaliar ampla e detalhadamente hábitos, tendências e práticas sexuais em mulheres, sendo um questionário simplificado para detectar possíveis portadores de dificuldades sexuais de diferentes níveis. A pontuação total do FSFI é a soma dos seis domínios, indentificando a presença ou ausência de disfunção sexual com base em um ponto de corte total de 26,55 , sendo o escore $\leq 26,55$ classificados como a presença de disfunção sexual e $>26$ um baixo risco de disfunção sexual. ${ }^{18,19}$ 
Para a realização do exame físico que foi composto pelo esquema PERFECT que objetivava avaliar a contração do MAP. As participantes ficaram em decúbito dorsal em posição ginecológica, cobertas por um lençol, utilizando luvas de vinil e gel lubrificante a base de água, e o examinador introduzia o dedo médio e indicador no canal vaginal. As participantes eram orientadas sobre como realizar a contração adequada da musculatura do assoalho pélvico MAP, no qual foi solicitado que a mulher contraísse o assoalho pélvico contra o dedo do avaliador (como se fosse segurar “o xixi”), sendo, nesse momento, realizada a graduação da atividade funcional do assoalho pélvico por meio do esquema PERFECT que foi desenvolvido e validado para avaliar a contração do MAP, composto por sete itens. O "P" (power/força) avalia a intensidade da contração na qual 0 é a ausência de resposta muscular e 5 contrações voluntária máxima da MAP, segundo a escala de Oxford modificada. O "E" (endurancel resistência) avalia quanto tempo à contração voluntária máxima (CVM) é mantida e sustentada em segundos, antes que haja uma perda de $35 \%$ ou mais da contração. O "R" (repetitions/repetições) avalia o número de repetições da contração sustentada (até 10 vezes) que a participante consegue realizar com duração satisfatória (5 segundos), com intervalo de 4 segundos entre cada contração. O "F” (fast/contrações rápidas) é avaliado após uma pequena pausa para repouso (1 minuto), ele avalia o nú- mero de CVM com duração de 1 segundo cada (até 10 contrações) ${ }^{20}$, tal mensuração deu-se por três vezes consecutivas na qual foi registrada a melhor performance, visando garantir o maior entendimento de como funciona o teste por parte da participante da pesquisa. Os dados foram inseridos em uma planilha eletrônica do programa $\mathrm{Mi}$ crosoft Excel, versão 2013. As análises dos dados foram realizadas pelo programa estatístico Epi Info ${ }^{\mathrm{\tau m}}$, sendo considerados: média, desvio padrão e percentual para as variáveis descritivas e quantitativas contínuas, para associação entre variáveis categóricas, utilizou-se o teste do qui-quadrado ( $\left.\chi^{2}\right)$ ou Exato de Fisher, quando necessário. A comparação entre as variáveis numéricas foi realizada pelo teste de Mann-Whitney.

\section{Resultados}

Participaram desta pesquisa $32 \mathrm{mu}$ lheres, com média de idade de 25,56 anos $(\mathrm{DP} \pm 6,35)$ sendo a mínima 19 e máxima 40 anos. Em maior percentual, declararam-se solteiras $(59,38 \%)$ e não possuíam filhos $(68,75 \%)$. Entre as mulheres que tinham filhos, o tipo de parto mais frequente foi o parto cesárea, ocorrendo em $71,88 \%$ das vezes. Em relação ao tempo da prática do CrossFit $^{\oplus}$, a média foi de 18,28 (DP $\left.\pm 12,38\right)$ meses e intervalo de tempo de 6 a 45 meses. Em relação à ocorrência de perda de urina durante a prática de $\mathrm{CrossFit}^{\oplus}$, houve queixa de incontinência em $75 \%$ das entrevistadas (Tabela 1). 
Tabela 1. Perfil sociodemográfico e incidência de perda de urina durante a prática do CrossFit ${ }^{\oplus}$.

\begin{tabular}{|c|c|c|}
\hline Variáveis & $\begin{array}{l}\text { Média } \\
\text { (Desvio } \\
\text { padrão) }\end{array}$ & N (\%) \\
\hline Idade (anos) & $\begin{array}{c}25,56 \pm \\
6,35\end{array}$ & - \\
\hline $\begin{array}{l}\text { Estado civil } \\
\text { Solteira } \\
\text { Casada } \\
\text { Divorciada/separada } \\
\text { Viúva } \\
\text { Não declarou }\end{array}$ & - & $\begin{array}{c}19 \\
(59,38 \%) \\
6 \\
(18,75 \%) \\
5 \\
(15,63 \%) \\
1(3,13 \%) \\
1(3,13 \%) \\
\end{array}$ \\
\hline $\begin{array}{l}\mathrm{N}^{\circ} \text { de Filho (s) } \\
0 \\
1 \\
2 \\
3\end{array}$ & - & $\begin{array}{c}17 \\
(53,13 \%) \\
7 \\
(21,88 \%) \\
4 \\
(12,50 \%) \\
4(12,50 \%)\end{array}$ \\
\hline $\begin{array}{l}\text { Tipo de parto } \\
\text { Cesárea } \\
\text { Vaginal }\end{array}$ & - & $\begin{array}{c}9 \\
(28,13 \%) \\
6 \\
(18,75 \%) \\
\end{array}$ \\
\hline $\begin{array}{l}\text { Tempo de prática } \\
\text { CrossFit }^{\oplus} \text { (meses) } \\
\end{array}$ & $\begin{array}{c}18,28 \pm \\
12,38 \\
\end{array}$ & - \\
\hline $\begin{array}{l}\text { Perda de urina } \\
\text { durante prática de } \\
\text { Crossfit }^{\oplus} \\
\text { Sim } \\
\text { Não }\end{array}$ & & $\begin{array}{c}24 \\
(75,00 \%) \\
8 \\
(25,00 \%)\end{array}$ \\
\hline $\begin{array}{l}\text { Qual(is) exercício(s) } \\
\text { Double under } \\
\text { Box jump } \\
\text { Medicine ball clean } \\
\text { Kettlebell swing }\end{array}$ & - & $\begin{array}{c}9 \\
(28,13 \%) \\
7 \\
(21,88 \%) \\
5 \\
(15,63 \%) \\
3(9,38 \%)\end{array}$ \\
\hline
\end{tabular}

$\mathrm{Na}$ página seguinte apresenta-se a $\mathrm{Fi}$ gura 1, exemplificando os exercícios citados pelas voluntárias como os que mais causavam perda de urina durante a execução do esporte e mais houveram correlação com as perdas urinárias, conhecidos como: $1^{\circ}$ Double Under com $(28,13 \%)$ que consiste em um pulo de corda, no qual a corda passa duas vezes por baixo dos pés da pessoa a cada salto; $2^{\circ}$ Box Jump (21,88\%), tal exercício é executado a partir de uma posição estática para um salto simultâneo de ambas as pernas sobre uma caixa de $75 \mathrm{~cm}$ de comprimento, $50 \mathrm{~cm}$ de largura e $60 \mathrm{~cm}$ de altura, o movimento é finalizado com o corpo em posição ortostática sobre a caixa; 3o Medicine-Ball Clean (15,63\%), esse exercício trata-se de um agachamento completo segurando uma medicine ball (bola revestida de couro, com variedade de tamanhos e pesos, geralmente variando de poucos centímetros de diâmetro, podendo ser do tamanho de uma bola de beisebol a tamanhos maiores como uma bola de basquetebol, o peso pode variar de $250 \mathrm{~g}$ até 50 $\mathrm{kg}$ ) o movimento é concluído quando se chega a uma extensão completa dos membros inferiores; $4^{\circ}$ Kettlebell Swing, com $(9,38 \%)$, para executá-lo é necessário segurar um kettlebell ou peso russo (bola de ferro fundido com uma alça) com ambas as mãos, saindo de uma altura abaixo do quadril até acima da cabeça em movimentos agis de extensão de quadril, embalando um trabalho corporal completo. ${ }^{21}$

Fonte: Dados da pesquisa, 2019. 

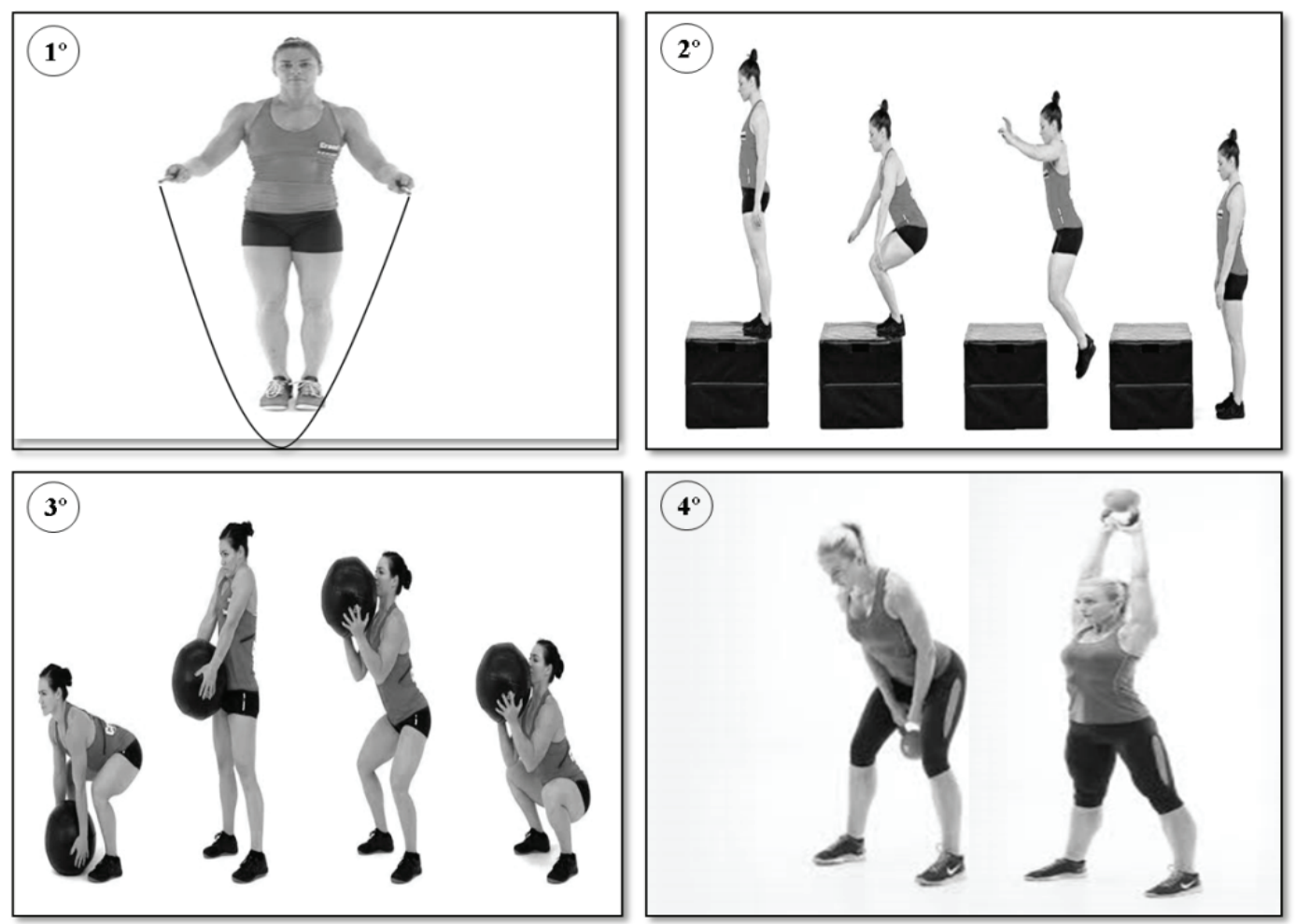

Figura 1 - Exercícios relacionados á perda de urina durante a prática do CrossFit ${ }^{\circledast}$.

Fonte: TELES, A.; AMIDANI, P., (2019).

Ao analisarmos as praticantes de CrossFit $^{\oplus}$ que relatam episódios de incontinência urinária durante o exercício, a média de idade foi de 26,25 anos (DP $\pm 6,76$ ) com o tempo de prática do esporte em 19,25 de meses (DP $\pm 10,65)$. Em relação aos tipos de parto e à perda de unira $77,78 \%$ das mulheres com incontinência que realizaram parto vaginal, observou-se também que a perda urinária foi a queixa mais recorrente em $80 \%$ das participantes que têm apenas um filho, isso se deve ao fato desta amostra conter um elevado número de mulheres uníparas, sendo seguida por $77,27 \%$ que não têm nenhum filho (Tabela 2).

A respeito dos resultados do esquema PERFECT representado pelos valores de média, observamos que o esquema obteve resultados baixos em todos os itens e que a funcionalidade do assoalho pélvico representa a função prejudicada. O Power (Força) obteve 2,84 (DP $\pm 0,72)$ tendo por valor de referência de 0 a 5 para avaliar a presença e a intensidade da contração muscular voluntária de acordo com a escala de Oxford modificada; Endurance (Resistência) 3,71 (DP \pm 1,57) sendo o parâmetro o tempo, em segundos da contração mantida e sustentada (no máximo de 10 segundos); Repetitions (Repetições) 3,53 (DP $\pm 0,98$ ) em que o padrão de normalidade avalia o número de repetições da contração sustentada (até 10 vezes) e Fast (Rapidez) 5,37 ( $\mathrm{DP} \pm 1,64)$, tendo o número de contração voluntária máxima com dura- 
Tabela 2. Exercícios mencionados com episódios de incontinência urinária durante o CrossFit $^{\circledast}$ e as variáveis: idade, tempo de prática (meses), tipos de parto e número de filhos.

\begin{tabular}{|c|c|c|c|c|c|c|}
\hline \multirow{2}{*}{\multicolumn{2}{|c|}{ Variável }} & \multicolumn{4}{|c|}{ Episódios de incontinência urinária durante o CrossFit ${ }^{\oplus}$} & \multirow[t]{2}{*}{ P-valor } \\
\hline & & $\begin{array}{c}\text { SIM } \\
\text { Media }\end{array}$ & $\mathrm{DP} \pm$ & $\begin{array}{l}\text { NÃO } \\
\text { Media }\end{array}$ & $\mathrm{DP} \pm$ & \\
\hline \multicolumn{2}{|c|}{ Idade } & 26,25 & 6,76 & 23,50 & 4,72 & 0,29 \\
\hline \multicolumn{2}{|c|}{$\begin{array}{c}\text { Tempo de prática do } \\
\text { esporte }\end{array}$} & 19,25 & 17,46 & 17,95 & 10,65 & 0,80 \\
\hline & & Frequência & No & Frequência & $\mathbf{N}^{\circ}$ & \\
\hline \multirow{2}{*}{$\begin{array}{c}\text { Tipos } \\
\text { de parto } \\
\end{array}$} & Cesárea & $66,67 \%$ & 4 & $33,33 \%$ & 2 & \multirow{2}{*}{0,86} \\
\hline & Vaginal & $77,78 \%$ & 7 & $22,22 \%$ & 2 & \\
\hline \multirow{4}{*}{$\begin{array}{l}\mathrm{N}^{\circ} \text { de } \\
\text { filhos }\end{array}$} & 0 & $76,47 \%$ & 13 & $23,53 \%$ & 4 & \multirow{4}{*}{0,81} \\
\hline & 1 & $80,00 \%$ & 8 & $20,00 \%$ & 2 & \\
\hline & 2 & $66,67 \%$ & 2 & $33,33 \%$ & 1 & \\
\hline & 3 & $50,00 \%$ & 1 & $50,00 \%$ & 1 & \\
\hline
\end{tabular}

Fonte: Dados da pesquisa, 2019.

ção de 1 segundo cada (até 10 contrações) como referência de regularidade de acordo com o esquema PERFECT. ${ }^{20}$ Ao analisar os dados obtidos, foi possível observar que todas as variáveis de medianas encontram-se consideravelmente baixas, como demonstradas na figura 2.

Quando associado à variável Power (Força) das praticantes que relataram incontinência urinária durante o CrossFit ${ }^{\oplus}, 41,67 \%$ das mulheres apresentaram grau de força 2 que representa: presença de contração de pequena intensidade, mas que se sustenta, segundo a escala de 0 a 5 de Oxford modificada. ${ }^{22}$
Em relação à análise da disfunção sexual encontrada na avaliação do questionário FSFI que é um questionário breve, autoaplicável, e que se propõe a avaliar a resposta sexual feminina em seis domínios: desejo sexual, excitação sexual, lubrificação vaginal, orgasmo, satisfação sexual e dor. Foram apresentadas 19 questões que avaliam a função sexual nas últimas quatro semanas. Para cada questão existe um padrão de resposta cujas opções recebem pontuação de 0 a 5 de forma crescente em relação à presença da função questionada. ${ }^{18,19}$ Os resultados foram: desejo sexual, média 3,68 (DP $\pm 1,49)$; 


\section{Avaliação Funcional do Assoalho Pélvico}

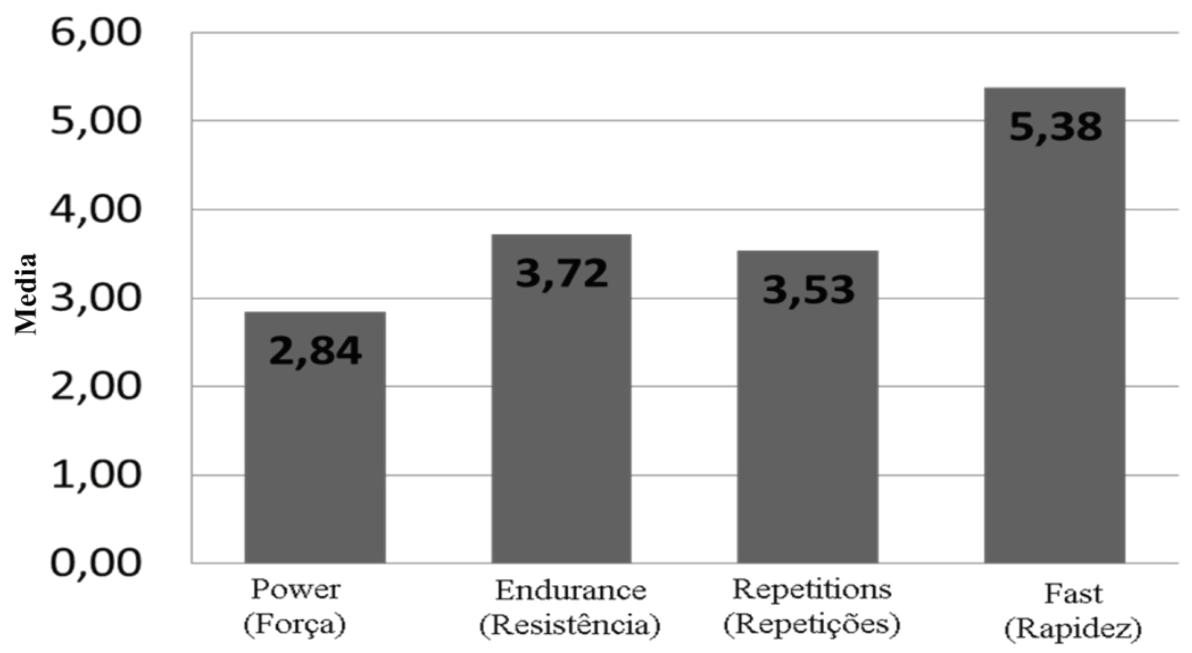

Figura 2. Gráfico referente à avaliação funcional do assoalho pélvico.

Fonte: Dados da pesquisa, 2019.
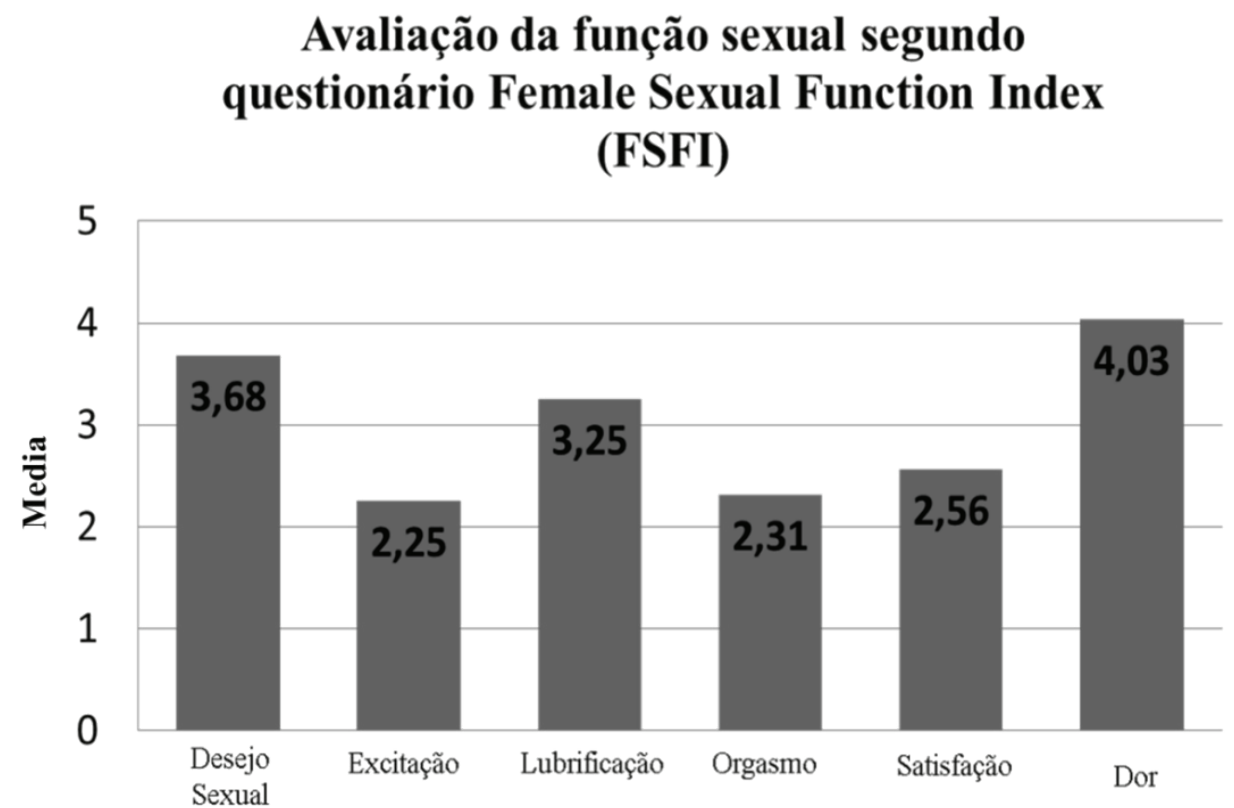

Figura 3. Gráfico referente à análise da disfunção sexual encontrados na avaliação do questionário FSFI.

Fonte: Dados da pesquisa, 2019. 
excitação, 2,25 (DP \pm 0,95); lubrificação de 3,25 ( $\mathrm{DP} \pm 1,24$ ); orgasmo, 2,31( $\mathrm{DP} \pm 1,06$ ); satisfação, 2,56(DP $\pm 1,04)$ e dor, 4,03(DP \pm 1,42). Os resultados dos domínios revelamse relativamente baixos, conforme descreve a figura 3.

Os dados referentes ao escore total do questionário FSFI apresentaram média de $18,15(\mathrm{DP} \pm 3,18)$ entre as entrevistadas. Segundo a pontuação de "corte" do questionário, valores $\leq 26$ referem-se a um alto risco de disfunção sexual. ${ }^{19}$

\section{Discussão}

Os resultados observados neste estudo indicaram que a prática do CrossFit ${ }^{\circledR}$ apresenta repercussões na função muscular do assoalho pélvico, houve queixa de incontinência urinária em $75 \%$ das entrevistadas, tais mulheres tiveram tempo médio de 19 meses de prática do esporte e a média de idade foi de 26 anos, revelando que até mesmo mulheres jovens podem ter sintomas de perda urinária quando submetidas a um estímulo desencadeante: no caso, o CrossFit ${ }^{\circ}$. Yang (2019) $)^{23}$, ao pesquisar 105 mulheres > 18 anos e com boa saúde, constatou que incontinência urinária pode ocorrer em mulheres jovens e fisicamente ativas durante $o$ exercício.

Em seus estudos ou pesquisas, Gephart et al (2018) ${ }^{24}$ e Yang et al (2019) ${ }^{23}$ ressaltam a relação direta entre o tipo de parto (vaginal) e o número de gestações (multíparas) somados aos efeitos do CrossFit ${ }^{\circledR}$ como fatores desencadeantes de IU. Contudo, no presente estudo, como mostra na Tabela 2, os altos índices de mulheres que têm apenas um filho apresentaram maior queixa de perda de urina, demostrando que independentemente da quantidade de filhos a modalidade já apresenta consequência direta sobre a MAP. Nesse sentido, é importante ressaltar que esses exercícios tendem a ser um fator de risco para o desenvolvimento da IU na mulher, tendo ou não relação com outros fatores.

Outro dado pertinente a ser considerado é a relação entre a paridade e a queixa de perda urinária durante a pratica do Cross$\mathrm{Fit}^{\circ}$. Entre as praticantes que disseram ter filhos, o parto cesáreo foi o mais prevalente $(71,88 \%)$, porém entre as que relataram perda de urina durante o esporte, o parto vaginal foi o de maior predomínio $(77,78 \%)$, isso se justifica pelo trabalho de parto e parto vaginal favorecem lesões nos tecidos moles pélvicos, das paredes vaginais, ruptura da fáscia endopélvica e dos nervos, essas estruturas representam importante fator na manutenção da continência urinária, além disso, o aumento de peso durante a gestação pode elevar o risco para o surgimento de IU. ${ }^{25,26}$ Nesse sentido, o presente estudo diverge do estudo de Almeida et al. (2015) ${ }^{11}$ realizado com mulheres de 18 a 49 anos que praticavam Jump, no qual, $66,7 \%$ das incontinentes realizaram partos cesáreos em relação ao parto vaginal. Em outro estudo realizado por meio de entrevista com 332 mulheres, os autores concluíram que a incontinência urinária de esforço atinge preferencialmente mulheres com antecedentes de algum tipo de parto, ainda que tenha sido cesáreo. ${ }^{27}$

A média de tempo de prática do Cros$\mathrm{sFit}^{\oplus}$ das incontinentes foi de 19,25 meses, superior à média das que declararam a não perda de urina durante o esporte, sendo de 
17,95 meses. Essas respostas são compatíveis aos achados de Antunes et al. $(2011)^{15}$, nos quais $54 \%$ da amostra realizaram a prática de atividades físicas por um período entre 1 a 5 anos, demonstrando essa relação entre o tempo de prática e o surgimento de queixas de IU em que essas mulheres sofrem sobrecarga constante no períneo pelo aumento da pressão intra-abdominal durante longos períodos de tempo.

Nesse contexto, o exercício físico, principalmente os de alta intensidade, é considerado um fator de risco para o surgimento de incontinência urinária de esforço em mulheres, visto que, durante o treinamento, a pressão intra-abdominal é aumentada, sobrecarregando a musculatura que compõe o assoalho pélvico, influenciando no mecanismo de continência. Durante o esforço o aumento da pressão intra-abdominal deve ser simultâneo e proporcional ao da pressão de fechamento uretral, porém quando não há o equilíbrio entre essas duas pressões, a pressão intra-abdominal se eleva o suficiente para permitir que a pressão intravesical exceda a pressão intra uretral, favorecendo a perda involuntária de urina. ${ }^{11,28,29}$

Yang et al. (2019) ${ }^{23}$, apontam que praticantes de CrossFit ${ }^{\circledast}$ relatam episódios de incontinência urinária durante o exercício. Segundo o autor, durante a prática do Cros$\mathrm{sFit}^{\circledast}, 80 \%$ das mulheres têm perda urinária geralmente associada a um tipo de exercício executado. No estudo, o autor estabeleceu 3 tipos de exercícios que causavam incontinência, (Top 3) consecutivos: o Double Under (47,7\%), o Jumping Rope (41,3\%) e o Box Jump (28,4\%), o que corroboram parcialmente com os achados desta pesquisa onde foram citados: Double Under,
Box Jump, Medicine-Ball Clean, Kettlebell Swing. Ao observarmos os exercícios citados, observaram-se várias equivalências na sua mecânica: o Double Under é caracterizado pelo pulo de corda, no qual a corda passa duas vezes de baixo dos pés a cada salto; o Box Jump consiste em partir de uma posição estática para um salto simultâneo de ambas as pernas sobre uma caixa com $75 \mathrm{~cm}$ de comprimento, $50 \mathrm{~cm}$ de largura e $60 \mathrm{~cm}$ de altura; o Medicine-Ball Clean trata-se um agachamento completo segurando uma medicine ball (bola pesada, revestida de couro, com peso aproximado do diâmetro dos ombros) o movimento é completado até chegar a uma extensão completa dos membros inferiores com os membros superiores estendidos, e o Kettlebell Swing segurando um kettlebell (peso russo) com ambas as mãos, saindo de uma altura abaixo do quadril até acima da cabeça em movimentos agis de extensão de quadril, embalando um trabalho corporal completo. ${ }^{21}$ Todos os exercícios apontados neste estudo envolvem saltos com reação máxima dos pês com o solo e/ou caixa, mudanças abruptas de movimentos, agachamentos suportando grandes cargas e movimentos cíclicos causando pressões constantes sobre o assoalho pélvico e aumentos abruptos da pressão abdominal que ocorrem constantemente durante a prática do CrossFit ${ }^{\circledast}$.

Movimentos de alta intensidade resultam em força de impacto sobre o assoalho pélvico de três a quatro vezes maiores que o peso corporal. Muitos autores destacam que a combinação entre aumento da pressão intra-abdominal exercida pelo tipo de exercício e a perda urinária, como fator desencadeante de disfunção do MAP. ${ }^{11,23,24,30}$ Diante 
disso, os exercícios citados têm como principais características os movimentos cíclicos e elevação de peso, sendo amplamente discutido como relação de causa e efeito para disfunção MAP. Bogéa et al (2018) ${ }^{31}$ ressalta ainda, que as atividades com saltos são as que apresentam maior possibilidade de causar condições favoráveis ao surgimento de incontinência urinaria de esforço.

$\mathrm{Na}$ avaliação dos músculos do assoalho pélvico, por meio do esquema PERFECT, ao analisar a amostra, observa-se um déficit na força muscular (média $=2,84$ ) que segundo a escala de Oxford modificada deve estar entre 0 a 5 , na qual 5 representa contração máxima do MAP. Ainda, nota-se redução da contração de fibras lentas ( édia $=3,72$ ) analisadas por meio do E (Endurance) correspondente ao tempo das contrações musculares mantidas e sustentadas (de no máximo dez segundos) e fibras rápidas (média $=5,38$ ) verificadas por meio do $\mathrm{F}$ (Fast) que significa o número de contrações rápidas (até 10 contrações) que, em ambas, quanto maior for o resultado melhor o desempenho muscular do MAP, contudo as variáveis referentes ao Power e Repetitions apontaram médias mais baixas demonstrando que as mulheres participantes de CrossFit ${ }^{\circ}$ avaliadas apresentaram uma redução de força muscular do MAP, consequentemente uma piora na capacidade de contração muscular, tornando assim deficitário o mecanismo de continência. Além de um déficit na resistência muscular implicando em uma sustentação insatisfatória, o que altera a função dos músculos do assoalho pélvico (gráfico 1 ).

Segundo Bø K (2004) ${ }^{32}$ no momento do exercício físico que geram aumento na pressão intra-abdominal não ocorrem à contração voluntária dos músculos do MAP. Segundo o mesmo estudo, mulheres praticantes de exercícios físicos nem sempre possuem o MAP mais forte do que as não praticantes.

O enfraquecimento pode contribuir de maneira efetiva no processo de IU. Korelo et al. $(2011)^{33}$ e Caldas et al. (2018) ${ }^{34}$ ainda comentam que os sintomas da IU melhoram principalmente através do ganho de força da MAP o que contribui para que possam resistir à pressão intra-abdominal que afeta indiretamente a pressão sobre a bexiga.

Ao analisar as variáveis que envolvem a função sexual por meio dos escores do FSFI (Gráfico 2), os domínios de excitação $(2,25)$ e orgasmos $(2,31)$ apresentaram as menores médias em relação à avaliação da função sexual. Para Piassarolli et al. $(2010)^{35}$ a avaliação da função sexual é muito complexa, pois a sexualidade é um fenômeno multidimensional, associado aos fatores biológicos, psicológicos, socioculturais e por determinantes interpessoais. Neste estudo não foi possível assegurar correlação direta entre os escores do FSFI e a prática do CrossFit ${ }^{\circ}$, esse fato pode ter ocorrido devido ao número reduzido de mulheres estudadas. No entanto, a função do MAP das mulheres dessa pesquisa condiz com o estudo populacional realizado na Holanda por Slieker-ten et al. (2009) ${ }^{36}$ que demonstrou que a fraqueza da musculatura do assoalho pélvico é um dos fatores de risco para as disfunções sexuais o que contribui para a diminuição orgástica, algo que também foi encontrado neste estudo.

No estudo do Piassarolli et al. $(2010)^{35} \mathrm{o}$ treinamento da musculatura do assoalho pélvico apresentou aumento da força e melhora significativa nos escores do questionário 
FSFI e nas queixas sexuais. Isso evidencia que há uma relação direta da força do assoalho pélvico com a resposta sexual feminina o que ratifica os resultados desta pesquisa.

\section{Conclusão}

A prática do CrossFit ${ }^{\oplus}$ demonstrou influenciar a funcionalidade da musculatura do assoalho pélvico em mulheres jovens com média de 1 ano e 7 meses de prática especialmente quando somados ao tipo de parto vaginal e ao maior número de gestações, esses fatores demostraram desencadear a IU durante a prática do esporte, além de favorecer a redução da força do MAP que tem influência direta na função sexual.

Embora o tamanho da amostra seja de um número reduzido de mulheres, os achados estão condizentes com os da literatura, em que mulheres que praticam atividades físicas de impacto relatam perdas urinárias, destacamos ainda há necessidade de novas pesquisas devido à escassez de estudos científicos referente à prática do Crossfit ${ }^{\circledR}$ e suas repercussões, investigando as especificidades do esporte e de suas praticantes, analisando outras variáveis que tenham vínculo com a prática esportiva e as disfunções da MAP.

\section{REFERÊNCIAS}

1. Vasconcelos CTM, Vasconcelos JA Neto, Bezerra LRP, Augusto KL, Karbage SAL, Frota IPR, et al. Disfunções do assoalho pélvico : perfil sóciodemográfico e clínico das usuárias de um ambulatório de uroginecologia. Revista Gestão \& Saúde. 2013; 4(1): 1484-98

2. Machado LS. Avaliação Funcional do Assoalho pélvico em Atletas e dua Relação com a Incontinencia Urinária [dissertação]. Porto Alegre: universidade federal de ciências da saúde de Porto Alegre (UFCSPA); 2017

3. Stein SR, Pavan FV, Nunes EFC, Latorre GFS. Entendimento da fisioterapia pélvica como opção de tratamento para as disfunções do assoalho pélvico por profissionais de saúde da rede pública. Rev. ciênc. méd. 2018; 27(2):65-72

4. Henkes DF, Fiori A, Carvalho JAM, Tavares KO, Frare JC. Incontinência urinária: o impacto na vida de mulheres acometidas e o significado do tratamento fisioterapêutico. Semina cienc. biol. saude. 2015; 36(2):45-56

5. Horst W, Silva JC. Prolapsos de órgãos pélvicos: revisando a literatura. Arq. Catarin Med. 2016; 45 (2): 91-101

6. Cândido EB, Giarolla L, Fonseca AMR, Monteiro MVC, Carvalho TS, Silva AL Filho. Conduta nos prolapsos genitais. Femina. 2012; 40 (2): 98-104

7. Mendonça CR, Amaral WN. Tratamento fisioterapêutico das disfunções sexuais femininas - revisão da literatura. Femina. 2011; 39 (3): 139-42

8. Antonioli RS, Simões D. Abordagem fisioterapêutica nas disfunções sexuais femininas. Rev Neurociênc. 2010; 18(2):267-74

9. Glisoi SFN, Girelli P. Importância da fisioterapia na conscientização e aprendizagem da contração da musculatura do assoalho pélvico em mulheres com incontinência urinária. Rev Bras Clin Med. 2011; 9(6):408-13

10. Franceschet J, Sacomori C, Cardoso FL. Força dos músculos do assoalho pélvico e função sexual em gestantes. Rev Bras Fisioter. 2009; 13(5):383-9 
11. Almeida MB, Barra AA, Saltiel F, Silva-Filho AL, Fonseca AM, Figueiredo EM. Urinary incontinence and other pelvic floor dysfunctions in female athletes in Brazil: A cross-sectional study. Scand J Med Sci Sports. 2016; 26(9):1109-16

12. Almeida MBA, Barra AA, Figueiredo EM, Velloso FSB, Silva AL, Monteiro MVC, et al. Disfunções de assoalho pélvico em atletas. Femina. 2011; 39(8):395-402

13. Weisenthal BM, Beck CA, Maloney MD, DeHaven KE, Giordano BD. Injury Rate and Patterns Among CrossFit Athletes. Orthop J Sports Med. 2014; 2(4):2325967114531177

14. Araujo MP, Parmigiano TR, Negra LGD, Torelli L, Carvalho CG, Wo L, et al. Avaliação do assoalho pélvico de atletas: existe relação com a incontinência urinária? Rev Bras Med Esporte. 2015; 21(6):442-6

15. Dominski FH, Siqueira TC, Serafim TT, Andrade A. Perfil de lesões em praticantes de CrossFit: revisão sistemática. Fisioter. Pesqui. 2018; 25(2):229-39

16. Antunes MB, Manso VMC, Andrade NVS. Análise dos sinais e sintomas da incontinência urinária de esforço em mulheres de 25 a 50 anos praticantes de atividades físicas em academias. Ensaios e Ciênc. 2011; 15(1):83-95

17. Soares VMN, Schor N, Tavares C. M. Vidas arriscadas: uma reflexão sobre a relação entre o número de gestações e mortalidade materna. Rev BrasCresc Desenvol Hum. 2008; 18 (3): 254-63

18. Pacagnella RC, Martinez EZ, Vieira EM. Validade de construto de uma versão em português do Female Sexual Function Index. Cad Saúde Pública. 2009; 25 (11): 2333-44

19. Wiegel M, Meston C, Rosen R. The female sexual function index (FSFI): cross-validation and development of clinical cutoff scores. J Sex Marital Ther. 2005; 31 (1): 1-20

20. Souza LM, Pegorare ABG, Christofoletti G, Barbosa SRM. Influência de um protocolo de exercícios do método Pilates na contratilidade da musculatura do assoalho pélvico de idosas não institucionalizadas. Rev. bras. geriatr. gerontol. 2017; 20 (4): 485-93

21. Teles A, Amidani P. Dicionário para Crossfit: nomes de movimentos, exercícios, expressões, siglas e equipamentos [Internet]. Brasília: Kamom Crossfit; 2017 [Acesso em: 2019 nov 04]. Disponível em: https://kamoncrossfit.com/dicionario-e-glossario-do-crossfit.

22. Caroci AS, Riesco MLG, Rocha BMC, Ventura LJ, Oliveira SG. Avaliação da força muscular perineal no primeiro trimestre da gestação. Rev. Latino-Am. Enfermagem. 2014; 22 (6): 893-901

23. Yang J, Cheng JW, Wagner H, Lohman E, Yang SH, Krishingner GA, et al. The effect of high impact crossfit exercises on stress urinary incontinence in physically active women. Neurourol Urodyn. 2019; 38(2):749-56

24. Gephart LF, Doersch KM, Reyes M, Kuehl TJ, Danford JM. Intraabdominal pressure in women during CrossFit exercises and the effect of age and parity. Proc (Bayl Univ Med Cent). 2018; 31 (3): 289-93

25. Machado BA, Santos RS, Leme APC, Silva MCM Júnior. Ocorrência de sintomas urinários no pós parto vaginal imediato em primíparas. Rev. Pesqui. Fisioter.2017; 7 (3): 359-68

26. Figueiredo EM, Lara JO, Cruz MC, Quintão DMG, Monteiro MVC. Perfil sociodemográfico e clínico de usuárias de serviço de fisioterapia uroginecológica da rede pública. Braz. J. Phys. Ther. 2008; 12 (2): 136-42

27. Borges JBR, Guarisi T, Camargo ACM, Gollop TR, Machado RB, Borges PCG. Incontinência urinária após parto vaginal ou cesáreo. Einstein (São Paulo). 2010; 8 (2): 192-6

28. Rios JL, Silva BA. Fisiopatologia da incontinência urinária de esforço: artigo de revisão. Revista Digital. Bueno Aires. 2010; 14 (140).

29. Santana AAC, Machado KDG, Henrichs MFB, Aguiar IO, Nunes EFC, Latorre GFS. Incontinência urinária em mulheres praticantes de Cross Fit. Arquivos de Ciências do Esporte. 2019; 7(3):119-22. 
30. CAETANO AS, TAVARES MCG, LOPES MHB. Incontinência urinária e a prática de atividades físicas. Rev Bras Med Esporte. 2007; 13(4): 270-4.

31. Bógea M, Gomes P, Dinucci A, Dutra F, Zaidan P. Incontinência urinária de esforço em mulheres praticantes de CrossFit ${ }^{\circledR}$ : um estudo transversal de prevalência. International Journal of Development Research. 2018; 8 (7): 21642-5

32. BO K. Urinary incontinence, pelvic floor dysfunction, exercise and sport. Sports Med. 2004; 34 (7): 451-64.

33. Korelo RIG, Kosiba CR, Grecco L, Matos RA. Influência do fortalecimento abdominal na função perineal, associado ou não à orientação de contração do assoalho pélvico, em nulíparas. Fisioter. Mov. 2011; 24(1):75-85

34. Caldas CAS, Mitidieri AMS. Crossfit e incontinência urinária de esforço em mulheres entre 18 e 45 anos. Saúde UniToledo. 2018; 2 (1): 104-17.

35. Piassarolli VP, Hardy E, Andrade NF, Ferreira NO, Osis MJD.Treinamento dos músculos do assoalho pélvico nas disfunções sexuais femininas. Rev Bras Ginecol Obstet. 2010; 32 (5): 233-40.

36. Slieker-ten Hove MC, Pool-Goudzwaard AL, Eijkemans MJ, Steegers-Theunissen RP, Burger CW, Vierhout ME. Vaginal noise: prevalence, bother and risk factors in a general female population aged 45-85. Int Urogynecol J Pelvic Floor Dysfunct. 2009; 20 (8): 905-11.

\section{DADOS DOS AUTORES}

\section{Ketiane Moura Bezerra}

Graduanda de fisioterapia do Centro Universitário do Estado do Pará (CESUPA), Belém/PA - Brasil. keti.ane@hotmail.com

\section{Rainara dos Santos Monteiro}

Graduanda de fisioterapia do Centro Universitário do Estado do Pará (CESUPA), Belém/PA - Brasil. rainarasantos3@gmail.com

\section{Thiffany da Costa da Costa Lima}

Graduanda de fisioterapia do Centro Universitário do Estado do Pará (CESUPA), Belém/PA - Brasil. thiffany220@gmail.com

\section{Rafaela Cordeiro de Macêdo}

Mestre e Docente do Centro Universitário do Estado do Pará (CESUPA), Belém/PA - Brasil. Rafaela_cmacedo@hotmail.com

Submetido em: 13-3-2021

Aceito em: 15-10-2021 